\title{
Triplet design as an intraocular lens for high myopia
}

\author{
C. González, L. Carretero, I. Pascual, and A. Fimia
}

\begin{abstract}
We present the possibility of using a triplet as an intraocular lens (IOL). The matrix method was used to calculate the power of this triplet for high myopia. The thickness of the lens, the distances between the edges of the IOL and the endothelium, the distances between the edges of the IOL and theiris, and the influence of axial displacement have been analyzed. We have also compared the size, the thickness, the power, the distances between the edges of the IOL and the endothelium, and the distances between the edges of the IOL and the iris to the typical concave-plane lens that is usually implanted in highly myopic eyes. As we can see, when the anterior chamber depth is normal, our triplet presents a better geometrical form than the concave-planelens.
\end{abstract}

Key words: Intraocular lens, matrix methods, optical properties, high myopia.

\section{Introduction}

There are several possible ways to correct high myopia: spectaclelenses, contact lenses, and intraocular lenses (IOL's). Each of these corrective systems has a series of advantages and disadvantages. In this paper we center on the analysis and design of the anterior chamber IOL's.

Recent research in the field of design has led us to the introduction of triplets as intraocular corrective systems in the anterior chamber.1,2 The advantages of these systems basically include the lighter weight of the lens, the ability to adapt the shape of the lens to the shape of the anterior chamber, and even the possibility of adding pharmaceuticals for therapeutic purposes to this type of IOL.

Prior to these types of solutions for high myopia, we had the Baikoff negative lens (concave plane), which has a high degree of implantation and which, when kept within certain geometric margins, provides excellent results in the correction of the afore mentioned ametropia. Here the shape, the power, and the tolerance levels of the implanted concaveplane lens are studied from a geometric optics perspective and within paraxial approximation, as is the

The authors are with the Laboratorio de Optica, Departamento Interuniversitario de Optica, Universidad deAlicante, Apartado 99, Alicante E03080, Spain.

Received 6 August 1993; revised manuscript received 14 September 1994.

0003-6935/95/162898-10\$06.00/0.

(c) 1995 Optical Society of America. triplet lens that we suggest as a solution for myopia magna.

The system that we propose is basically a triplet formed by two lenses in the shape of a meniscus that leaves the central zone of the triplet empty. Because of the difference in indices the geometric shape is inverted; this improves the edge thicknesses, and therefore the system can be adapted to the shape of the anterior chamber (see Fig. 1).

Sections 2 and 3 are dedicated to the calculation of the power of this type of lens. These cal culations are done by use of matrix methods.3,4 In Section 4 we analyze the thickness and geometry of the lenses, and in Section 5 we compare these designs with those of the concave-plane lens and simultaneously apply our matrix method for power calculation to this lens. In Section 6 we study the influence axial displacement has on the calculated power.

\section{Matrix Calculations}

Of the several parameters that should be used to define a lens, the most important one for the IOL is the emmetropic power.

Different methods and formulas exist that can be used to calculate the power of IOL's, such as the lines of regression or geometrical optics methods, ${ }^{5}$ or the one we use here, matrix methods of geometrical optics. In Appendix A we include the shape of the refraction and translation matrices that we use in this study, as well as the definition of the different parameters that intervene in them.

We take into account all of the data from the unaccommodated Le Grand theoretical eye, ${ }^{6}$ except 




Fig. 1. Schematic of a phakic eye.

anterior and posterior radii of curvature of the cornea and the axial length, because we assume that the eyes that are being analyzed are myopic. This system eye can be made emmetropic if we place a lens in the anterior chamber in such a way that its power corrects the ametropia and distant objects form an image on the retina.

Therefore, given the refraction and translation matrices and taking into account that we are working with an eye such as the one represented in Fig. 2, we find that the matrix associated with the eye as an optical system is equal to the product of the matrices associated with the cornea, the IOL, and the crystalline lens. Likewise, we should keep in mind the translations between each of the aforementioned elements. Mathematically this is expressed as follows:

$$
M=M_{C} T_{L C} M_{L} T_{C O L} M_{C O},
$$

where $M_{C O}$ is the matrix of the cornea, $M_{L}$ is the matrix of the $\mathrm{IOL}$, and $\mathrm{M}_{\mathrm{C}}$ is the matrix of crystalline, and that at the same time each of these is made up of a series of matrices that will be defined later in the paper. The $T$ matrices are the translations between the different surfaces of the systems that have been defined.

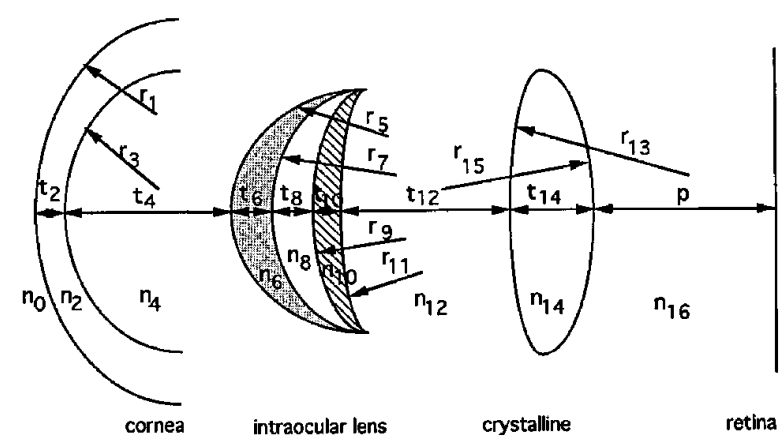

Fig. 2. Detailed scheme of the analyzed eye system.
A. Cornea Matrix

Considering that the cornea is made up of two surfaces separated by a thickness, $t_{2}$, we see that the cornea matrix $\left(\mathrm{M}_{\mathrm{CO}}\right)$ is the product of three matrices:

$$
M_{\mathrm{CO}}=\left[\begin{array}{cc}
1 & 0 \\
\frac{P_{3}}{\mathrm{n}_{4}} & \frac{\mathrm{n}_{2}}{\mathrm{n}_{4}}
\end{array}\right]\left[\begin{array}{cc}
1 & -\mathrm{t}_{2} \\
0 & 1
\end{array}\right]\left[\begin{array}{cc}
1 & 0 \\
\frac{\mathrm{P}_{1}}{\mathrm{n}_{2}} & \frac{\mathrm{n}_{0}}{\mathrm{n}_{2}}
\end{array}\right]=\left[\begin{array}{ll}
\mathrm{a}_{\mathrm{CO}} & \mathrm{b}_{\mathrm{CO}} \\
c_{\mathrm{CO}} & \mathrm{d}_{\mathrm{CO}}
\end{array}\right] .
$$

All of these parameters are defined in Appendix B, and we substitute both the indices and the thickness with their corresponding values. The anterior and posterior powers of the cornea are considered parameters, as normally one of the pieces of data we use at the outset is the power of the cornea as a whole (total corneal power). Hence $\mathrm{a}_{\mathrm{CO}}, \mathrm{b}_{\mathrm{CO}}, \mathrm{c}_{\mathrm{CO}}$, and $\mathrm{d}_{\mathrm{CO}}$ are given by

$$
\begin{aligned}
& \mathrm{a}_{\mathrm{CO}}=\frac{-0.00055}{1.3771} \mathrm{P}_{1}+1, \\
& \mathrm{~b}_{\mathrm{CO}}=\frac{-0.00055}{1.3771}, \\
& \mathrm{c}_{\mathrm{CO}}=\frac{-0.00055}{1.3374 \times 1.3771}\left(\mathrm{P}_{3} \mathrm{P}_{1}\right)+\frac{\mathrm{P}_{3}+\mathrm{P}_{1}}{1.3374}, \\
& \mathrm{~d}_{\mathrm{CO}}=\frac{-0.00055 \mathrm{P}_{3}}{1.3374 \times 1.3771}+\frac{1}{1.3374} .
\end{aligned}
$$

In clinical practice it is not possible to measure the radius of curvature of the posterior surface of the cornea. It is possible, however, to estimate its value from the measurement of the radius of curvature of the anterior surface of the cornea. ${ }^{3}$

\section{B. Crystalline Matrix}

The crystalline matrix $\left(\mathrm{M}_{\mathrm{C}}\right)$ can also be found by multiplication of the refraction and translation matrices of its anterior and posterior surfaces. Therefore we can say that analogously the crystalline matrix is

$$
M_{C}=\left[\begin{array}{ll}
a_{C} & b_{C} \\
c_{C} & d_{C}
\end{array}\right],
$$

where $a_{c}=0.977183, b_{c}=-0.0037673, c_{c}=16.3028$, and $d_{c}=0.961569$. To determine these coefficients we use the unaccommodated Le Grand theoretical eye.

C. Intraocular Lens Matrix

As we mentioned above, the most widely accepted solution in the literature is the implantation of a concave-plane lens, which is simply a negative concaveplanelens. The optical results are very good but this lens involves a certain risk to the integrity of the corneal endothelium. 7,8 There are several papers on the damage done by concave-plane lenses to the corneal endothelium, ${ }^{9-12}$ in which significant morpho- 
logic endothel ial changes were noted al ong the edge of the optic lens. Around dark zones, which appeared acellular, endothelial cells were enlarged, deformed, and separated. These changes might have been produced by intermittent contact between the endothelium and the lens. The edge thickness of the optic lens (aproximately $1 \mathrm{~mm}$ for high power) and the high vault of the IOL (to avoid contact with the crystalline lens) are probably the reasons for contact between the edge of the optic lens and the endothelium, particularly during eye rubbing.

The implantation of concave-plane IOL's is al most completely unacceptable because of the endothelial alterations they produce. However, research on the implantation of IOL's in phakic myopic eyes should not be abandoned because this technique produces a more accurate, predictable, and stable cor rection than the corneal refractive surgery techniques of myopic keratomileusis and epi keratoplasty. ${ }^{12}$

The solution that we offer and analyze in this paper is to consider the use of triplets that have an air chamber in the middle. The result is that even though we use positive lens geometry (the edge thickness smaller than the central thickness), the system works as a negative lens. Figure 2 shows the overall problem. The great number of parameters that come into play are clearly seen here, and this complicates the use of our system.

Therefore, we can say that the matrix associated with the IOL $\left(M_{L}\right)$ would be the product of the seven matrices associated with each of the translations and refractions that are produced on the different surfaces of the lens. These can be expressed as

$$
\begin{gathered}
M_{L}=\left[\begin{array}{cc}
1 & 0 \\
\frac{P_{11}}{n_{12}} & \frac{n_{10}}{n_{12}}
\end{array}\right]\left[\begin{array}{cc}
1 & -t_{10} \\
0 & 1
\end{array}\right]\left[\begin{array}{cc}
1 & 0 \\
\frac{P_{9}}{n_{10}} & \frac{n_{8}}{n_{10}}
\end{array}\right]\left[\begin{array}{cc}
1 & -t_{8} \\
0 & 1
\end{array}\right] \\
{\left[\begin{array}{cc}
1 & 0 \\
\frac{P_{7}}{n_{8}} & \frac{n_{6}}{n_{8}}
\end{array}\right]\left[\begin{array}{cc}
1 & -t_{6} \\
0 & 1
\end{array}\right]\left[\begin{array}{cc}
1 & 0 \\
\frac{P_{5}}{n_{6}} & \frac{n_{4}}{n_{6}}
\end{array}\right]=\left[\begin{array}{cc}
a_{L} & b_{L} \\
c_{L} & d_{L}
\end{array}\right] .}
\end{gathered}
$$

All of these parameters are defined in Appendix B. As we can see, we have maintained all of the variables that form part of the calculation in order to be able to influence the design of the lens and the calculation of its power.

\section{Power Calculations}

It is obvious that given the number of variables that we used in the calculation, initially it does not seem to make sense to present an equation associated with the lens power. Figure 3 shows the flow diagram associated with the numerical calculation done by computer, in which we determined the power of the IOL that should be implanted from the axial length of the eye, the total corneal power, and the lens position. The DERIVE program was used to multiply the matrices.

To calculate the power of the emmetropic lens implant, we had to meet the final condition that the

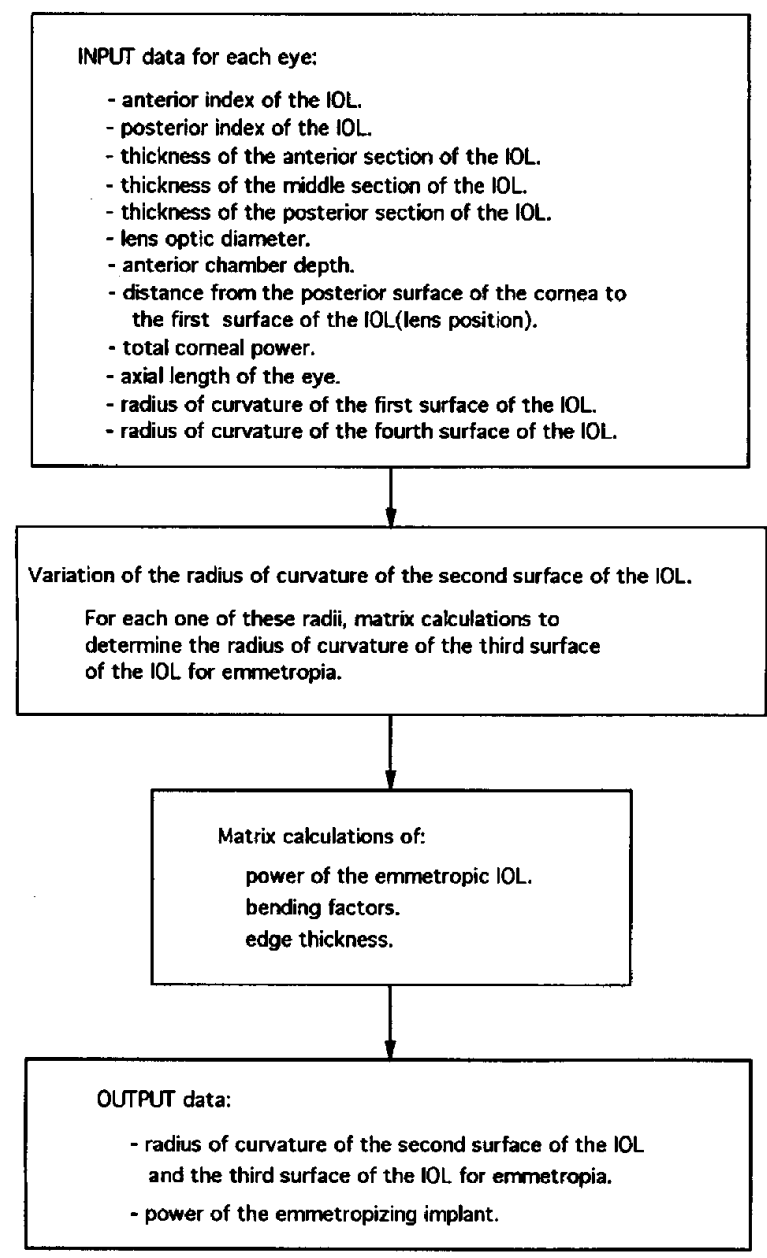

Fig. 3. Flow diagram of the program designed to carry out the IOL calculations.

posterior frontal distance of the eye system $\left(\mathrm{S}_{\mathrm{F}^{\prime}}{ }^{\prime}\right)$ be equal to the distance from the posterior crystalline surface to the retina $(p)$. The $S_{F}{ }^{\prime}$ distance is obtained from the total matrix of the eye:

$$
M=M_{C} T_{L C} M_{L} T_{C O L} M_{C O}=\left[\begin{array}{ll}
A & B \\
C & D
\end{array}\right] .
$$

Therefore this required condition is

$$
\mathrm{S}_{\mathrm{F}}{ }^{\prime}=\frac{\mathrm{A}}{\mathrm{C}}=\mathrm{p} .
$$

So that the shape of the lens adapts as well as possible to the shape of the anterior chamber, we considered the foll owing to be true for our design: $r_{5}=r_{3}$ and $r_{11}$ $=r_{13}$ (see Fig. 2). Once these values were established, Eq. (10) was used to find the value of $\mathrm{P}_{9}$ as a function of $P_{7}$.

One very important aspect of our design is the fact that we left the powers of the anterior and posterior surfaces of the empty zone as free parameters in our calculations so that we could obtain a set of lenses that could both make the eye emmetropic and have different bending factors and therefore influence the 
image quality. The equation of the power of the IOL can be determined by

$$
\mathrm{P}_{1 \mathrm{OL}}=\mathrm{n}_{12} \mathrm{C}_{\mathrm{L}},
$$

where $c_{\mathrm{L}}$ is one of the terms of the IOL matrix [Eq. (8)]. It makes no sense for us to give the complete equation, given the large number of variables that intervene.

The study of the calculation of powers of this IOL triplet was done for 12 theoretic eyes with their respective axial lengths, which ranged from 27 to 33 $\mathrm{mm}$, and their respective total corneal power, which ranged from 42 to $48 \mathrm{D}$ (where $\mathrm{D}$ is for diopter). These cover al most the entire range of possible cases of high myopia. Table 1 shows these 12 cases and their corresponding IOL powers that make myopic eyes emmetropic. E ach of these results is really a set of possible lenses. For example, Fig. 4 shows the variation in radii $r_{7}$ and $r_{9}$, which is explained by the fact that when the shape of the lens is changed, the placement of its principal image planes also changes; therefore, so does its power. Equation (10) always ensures that all of the lenses represented in Fig. 4 make the myopic eye emmetropic. From the point of view of design, one important aspect is to consider the relationship that exists between the calculated data and the real acceptable values for the manufacturing of these lenses. For all of the possible values of $r_{7}$ and $r_{9}$, the only ones that will be considered here are those that have an adequate minimum edge thickness.

\section{Edge Thickness}

Of all of the possible lenses that can be obtained by use of this calculation program, we should choose those that have the lowest possible thickness given the geometric conditions of the anterior chamber. Throughout our study we assume that the minimum thickness of a poly(methyl methacrylate) sheet is $0.125 \mathrm{~mm}$, and we do not consider any thickness below this. The reason for this minimum is to ensure the mechanical stability of the triplet. If we look at Fig. 5, we can see that a relationship exists among edge thickness, central thickness, the anterior sagitta, and the posterior sagitta of the lens. Edge thickness $(\mathrm{ET})$ is calculated as

$$
\mathrm{ET}=\mathrm{CT}-\mathrm{S}_{1}+\mathrm{S}_{2}
$$

where CT is central thickness, $\mathrm{S}_{2}$ is the posterior sagitta, and $\mathrm{S}_{1}$ is the anterior sagitta.

Table 1. Calculations of Triplet IOL Power for Emmetropia for 12 Theoretical Eyes

\begin{tabular}{cccc}
\hline & \multicolumn{3}{c}{ Axial Length $(\mathrm{mm})$} \\
\cline { 2 - 4 } $\begin{array}{c}\text { Total Corneal } \\
\text { Power }(\mathrm{D})\end{array}$ & 27 & 30 & 33 \\
\hline 42 & -6.93 & -13.39 & -18.53 \\
44 & -9.20 & -15.51 & -20.64 \\
46 & -11.49 & -17.72 & -22.77 \\
48 & -13.72 & -20.11 & -25.23 \\
\hline
\end{tabular}

$$
-\circ-r_{7}(\mathrm{~m}) \quad \longrightarrow \mathrm{r}_{9}(\mathrm{~m})
$$

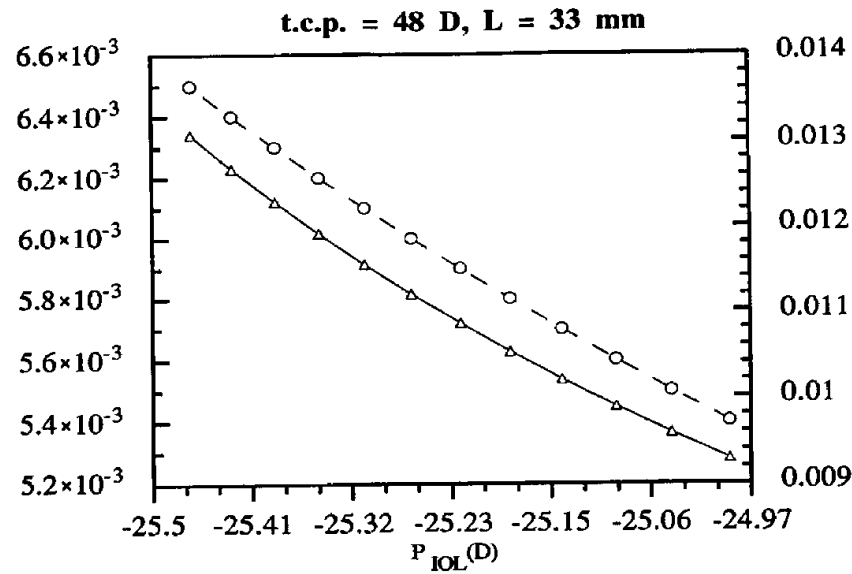

Fig. 4. Graph of the variation in $r_{7}$ and $r_{9}$ for one emmetropic power (t.c.p., total corneal power; L, axial length).

A lens is a system given for two diopters. Using el ementary Pythagorean geometry, we can obtain the expression of the sagitta $(\mathbf{S})$ for each spherical diopter:

$$
\mathrm{S}=|\mathrm{r}|-\left(\mathrm{r}^{2}-\mathrm{h}^{2}\right)^{1 / 2},
$$

where $r$ is the radius of curvature of the diopter and $h$ is the height for which we want to calculate the sagitta; in this case $h$ is half of the diameter of the optic lens $(\phi=2 \mathrm{~h}){ }^{13}$ In our case the IOL is made up of three meniscus lenses, and the edge thickness of each will be obtained from the data on the radii of the lens, their central thickness, and their diameter (we

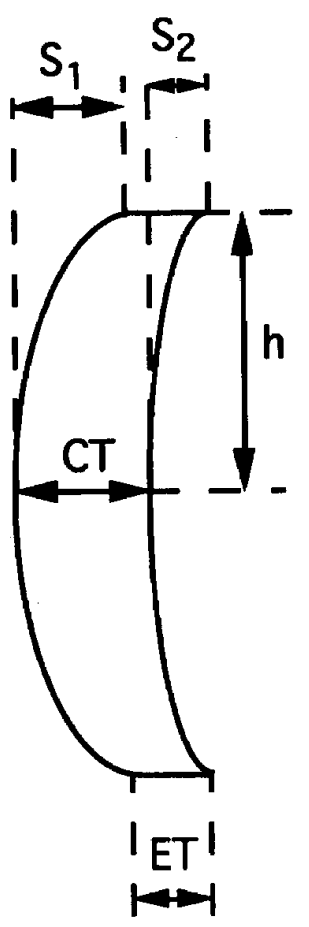

Fig. 5. Relationship between central thickness, edge thickness, anterior sagitta, and posterior sagitta for a meniscus.

1 June 1995 / Vol. 34, No. 16 / APPLIED OPTICS 
Table 2. Calculations of the Central and Edge Thicknesses of Each Lens of the Triplet for the 12 Theoretical Eyes

\begin{tabular}{|c|c|c|c|c|c|c|c|c|}
\hline \multirow{2}{*}{$\begin{array}{c}\text { Power } \\
\text { (D) }\end{array}$} & \multicolumn{3}{|c|}{ Edge Thickness (mm) } & \multirow{2}{*}{$\begin{array}{c}\text { Total Edge } \\
\text { Thickness }(\mathrm{mm})\end{array}$} & \multicolumn{3}{|c|}{ Central Thickness (mm) } & \multirow{2}{*}{$\begin{array}{r}\text { Total Central } \\
\text { Thickness }(\mathrm{mm}\end{array}$} \\
\hline & Anterior & Middle & Posterior & & Anterior & Middle & Posterior & \\
\hline-6.93 & 0.126 & 0.095 & 0.146 & 0.367 & 0.200 & 0.250 & 0.175 & 0.625 \\
\hline-13.39 & 0.127 & 0.027 & 0.138 & 0.292 & 0.150 & 0.250 & 0.150 & 0.550 \\
\hline-18.53 & 0.125 & 0.024 & 0.143 & 0.292 & 0.125 & 0.300 & 0.125 & 0.550 \\
\hline-9.20 & 0.130 & 0.055 & 0.135 & 0.320 & 0.200 & 0.250 & 0.175 & 0.625 \\
\hline-15.51 & 0.128 & 0.038 & 0.130 & 0.295 & 0.150 & 0.300 & 0.150 & 0.600 \\
\hline-20.64 & 0.129 & 0.034 & 0.132 & 0.295 & 0.125 & 0.350 & 0.125 & 0.600 \\
\hline-11.49 & 0.129 & 0.015 & 0.128 & 0.272 & 0.200 & 0.250 & 0.175 & 0.625 \\
\hline-17.72 & 0.131 & 0.049 & 0.142 & 0.322 & 0.175 & 0.350 & 0.150 & 0.675 \\
\hline-22.77 & 0.124 & 0.043 & 0.130 & 0.297 & 0.125 & 0.400 & 0.125 & 0.650 \\
\hline-13.72 & 0.130 & 0.025 & 0.141 & 0.296 & 0.225 & 0.300 & 0.175 & 0.700 \\
\hline-20.11 & 0.133 & 0.008 & 0.130 & 0.271 & 0.200 & 0.350 & 0.125 & 0.675 \\
\hline-25.23 & 0.139 & 0.003 & 0.154 & 0.296 & 0.175 & 0.400 & 0.125 & 0.700 \\
\hline
\end{tabular}

have considered a diameter of $6 \mathrm{~mm}$ ). Of all the possible IOL's that make a specific eye emmetropic, we can choose only those that have an appropriate edge thickness. Table 2 shows the optimal central and edge thicknesses for each of the lenses that make up the triplet for all 12 cases studied.

\section{Comparison of the Triplet and} Concave-Plane Lenses

By using a calculation program we have been able to analyze the cases of cornea power and axial length that are shown in Table 1. We assumed that we were dealing with myopic eyes. As we can see, the variations in lens power ranges from $-6 \mathrm{D}$ to $-25 \mathrm{D}$.

The calculation program allows us to evaluate both the curvature radii and the central thickness of each of the lenses in the triplet $\left(\mathrm{T}_{\mathrm{j}}\right)$. Moreover, by imposing the aforementioned conditions for edge thickness, we can calculate its value $\left(E_{\mathrm{j}}\right)$ for each case (Table 2 ). The total edge thickness of the IOL is one of the most important factors in deciding if an IOL can be implanted in phakic myopic eyes.

Figure 6 shows the evolution of the total edge and central thicknesses for the lens powers we analyzed.

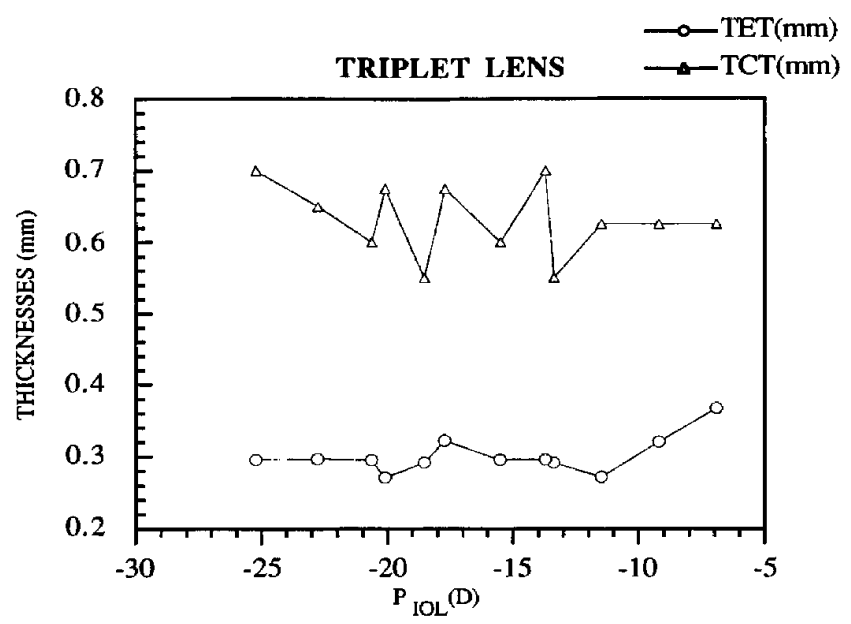

Fig. 6. Graph of edge and central thicknesses as a function of the corrective power of the triplet lens (TET, total edge thickness; TCT, total central thickness).
As we can observe, both the central and edge thicknesses evolve irregularly because the data we included correspond to lenses with optimized values. In this graph we must highlight that the edge thickness is approximately $0.3 \mathrm{~mm}$ and the central thickness is $0.7 \mathrm{~mm}$ (in the worst of the cases presented). These values are significant if we keep in mind that the anterior chamber has limited dimensions. ${ }^{6}$ According to several authors, ${ }^{14,15}$ the anterior chamber depth (ACD) can be predicted by use of a combination of the corneal curvature and the axial length. Other authors have not found any correlation between the amount of myopia and the depth of the anterior chamber. ${ }^{11}$ TheACD is an ocular dimension that can be directly measured for each eye, and therefore each eye has its own ACD value. Because in this paper we carry out a theoretical analysis, we used the same ACD value of $3.05 \mathrm{~mm}$ (Le Grand theoretical eye) for all cases. This ACD is the distance from the posterior vertex of the cornea to the anterior surface of the crystalline, measured along the eye's optical axis.

In designing the new triplet IOL that we proposed, we tried to keep the edge thickness as minimal as possible to avoid contact between the lens and the corneal endothelium. Therefore, it is important to know the dimensions of the peripheral anterior chamber in order to analyze the distances between the edge of the lens and the endothelium. If we take into account the models of the eye that Holladay et al. ${ }^{14}$ and Retzlaff et al. ${ }^{15}$ proposed in which the cornea is considered a section of a sphere, the base of which forms a plane at the level of the iris, then we can calculate the dimension of the peripheral anterior chamber for a specific height, $h$, by applying the formula for the sagitta [E q. (13)] for this height and by taking into account the ACD measured al ong the eye's optical axis as well. Given that the diameter of the IOL was $6 \mathrm{~mm}$, we should study the peripheral anterior chamber space at a height of $3 \mathrm{~mm}$. By applying the formula for the sagitta [Eq. (13)] we take into account what Holladay said about using the radius, $r$, in the calculations ${ }^{14,16:}$ "To take into account the asphericity of the cornea and the shorter 
Table 3. Calculations of the Peripheral ACD at a Height of $3 \mathrm{~mm}$ for the Four Total Corneal Powers Studied

\begin{tabular}{cc}
\hline Total Corneal Power $(\mathrm{D})$ & Peripheral ACD $(\mathrm{mm})$ \\
\hline 42 & 2.457 \\
44 & 2.426 \\
46 & 2.394 \\
48 & 2.375 \\
\hline
\end{tabular}

radius of curvature of the posterior surface of the cornea, compared with the anterior radius of curvature, one uses the anterior corneal radius $\left(r_{1}\right)$ rather than the posterior corneal radius $\left(r_{3}\right)$." However, if $r_{1}<7 \mathrm{~mm}$ then $r_{1}=7 \mathrm{~mm}$. In Table 3 we show the values of the peripheral anterior chamber size (distance from the posterior cornea to the iris) at a height of $3 \mathrm{~mm}$ for the four total corneal powers studied.

We al so analyzed the distances from the edge of the lens to the iris and to the corneal endothelium. We even found the percentage of the peripheral anterior chamber that the edge of the lens occupies. These data are shown in Table 4 for each of the 12 cases studied.

To compare the results, we carried out a calculation of the values of the lens power, the edge thickness, and the central thickness for the concave-planelenses that corresponded to the 12 theoretical eyes previously proposed. In Table 5 we give the results of our calculations, in which the minimal central thickness used was always $0.125 \mathrm{~mm}$ and the diameter was 6 $\mathrm{mm}$. In Fig. 7 we also show the results for edge and central thicknesses. It is quite clear that the central thickness is al ways the same. However, edge thickness increases when the absolute value of the lens power increases, reaching a value close to $1 \mathrm{~mm}$. If we try to decrease this value, we end up with a decrease in the usable optic diameter and hence a decrease in the active optic correction zone. When decreasing the optic zone we must keep in mind that if the lens's optic diameter is smaller than the pupil diameter, then vision problems will appear such as
Table 5. Calculations of Lens Power, Radius of Curvature, and Central and Edge Thicknesses of the Concave-Plane IOL for the 12 Theoretical Eyes

\begin{tabular}{ccrrcc}
\hline $\begin{array}{c}\text { Total } \\
\text { Corneal } \\
\text { Power }(\mathrm{D})\end{array}$ & $\begin{array}{c}\text { Axial } \\
\text { Length } \\
(\mathrm{mm})\end{array}$ & $\begin{array}{r}\text { Radii } \\
(\mathrm{mm})\end{array}$ & $\begin{array}{c}\text { Power } \\
(\mathrm{D})\end{array}$ & $\begin{array}{c}\text { Total Edge } \\
\text { Thickness } \\
(\mathrm{mm})\end{array}$ & $\begin{array}{c}\text { Total Central } \\
\text { Thick- } \\
\text { ness }(\mathrm{mm})\end{array}$ \\
\hline \multirow{4}{*}{42} & 27 & -21.05 & -7.25 & 0.340 & 0.125 \\
& 30 & -11.09 & -13.76 & 0.539 & 0.125 \\
& 33 & -8.02 & -19.02 & 0.707 & 0.125 \\
44 & 27 & -15.99 & -9.54 & 0.409 & 0.125 \\
& 30 & -9.50 & -16.06 & 0.611 & 0.125 \\
4 & 33 & -7.16 & -21.32 & 0.784 & 0.125 \\
& 27 & -12.87 & -11.86 & 0.480 & 0.125 \\
& 30 & -8.31 & -18.37 & 0.686 & 0.125 \\
48 & 33 & -6.46 & -23.63 & 0.864 & 0.125 \\
& 27 & -10.76 & -14.18 & 0.552 & 0.125 \\
& 30 & -7.37 & -20.70 & 0.763 & 0.125 \\
& 33 & -5.88 & -25.96 & 0.948 & 0.125 \\
\hline
\end{tabular}

glare and halos of colors. The following pupil size can be considered typical: for the eye in total darkness, $7.6 \mathrm{~mm}$ at age $10,6.2 \mathrm{~mm}$ at age 45 , and $5.2 \mathrm{~mm}$ at age 80; for the light-adapted eye, $4.8 \mathrm{~mm}$ at age 10 , $4.0 \mathrm{~mm}$ at age 45 , and $3.4 \mathrm{~mm}$ at age $80 .{ }^{17}$ We also analyzed the distance from the edge of the lens to the iris and to the corneal endothelium for the concaveplane lens. We found the percentage of the peripheral anterior chamber that the edge of the lens occupies. These data are shown in Table 4 together with the data on the triplet lens.

As we already said, in all cases analyzed except for that in which the lens had a power of $-6.93 \mathrm{D}$, the thickness of the edge of the triplet lens is less than that of the concave-plane lens (Tables 2 and 5). This decrease is very significant if we take into account the small dimensions of the peripheral anterior chamber (Table 3). The edge thickness of a triplet lens is only between $11 \%$ and $15 \%$ of the dimension of the peripheral anterior chamber (Table 4), and that leaves sufficient space so that no contact is made between the edges of the lens and the structures that surround

Table 4. Percentage of the Total Edge Thickness of the IOL in Relation to the Peripheral ACD and Distances from the Edges of the IOL to the Iris and to the Cornea at a Height of $3 \mathrm{~mm}$ for the 12 Theoretical Eyes

\begin{tabular}{|c|c|c|c|c|c|c|c|}
\hline \multirow{2}{*}{$\begin{array}{c}\text { Total Corneal } \\
\text { Power }(\mathrm{D})\end{array}$} & \multirow{2}{*}{$\begin{array}{c}\text { Axial } \\
\text { Length }(\mathrm{mm})\end{array}$} & \multicolumn{3}{|c|}{ Triplet Lens } & \multicolumn{3}{|c|}{ Concave-Plane Lens } \\
\hline & & $\%$ & $\mathrm{I}-\mathrm{E}^{\mathrm{a}}(\mathrm{mm})$ & $C-E^{b}(m)$ & $\%$ & $\mathrm{I}-\mathrm{E}^{\mathrm{a}}(\mathrm{m})$ & $C-E^{b}(m)$ \\
\hline & 27 & 15 & 0.761 & 1.329 & 14 & 1.463 & 0.655 \\
\hline \multirow[t]{3}{*}{42} & 30 & 12 & 0.799 & 1.367 & 22 & 1.463 & 0.456 \\
\hline & 33 & 12 & 0.799 & 1.367 & 29 & 1.463 & 0.288 \\
\hline & 27 & 13 & 0.761 & 1.344 & 17 & 1.463 & 0.554 \\
\hline \multirow[t]{3}{*}{44} & 30 & 12 & 0.774 & 1.357 & 25 & 1.463 & 0.352 \\
\hline & 33 & 12 & 0.774 & 1.357 & 32 & 1.463 & 0.179 \\
\hline & 27 & 11 & 0.761 & 1.361 & 20 & 1.463 & 0.451 \\
\hline \multirow[t]{3}{*}{46} & 30 & 13 & 0.736 & 1.336 & 29 & 1.463 & 0.245 \\
\hline & 33 & 12 & 0.749 & 1.348 & 36 & 1.463 & 0.067 \\
\hline & 27 & 13 & 0.724 & 1.355 & 23 & 1.463 & 0.360 \\
\hline \multirow[t]{2}{*}{48} & 30 & 11 & 0.736 & 1.368 & 32 & 1.463 & 0.149 \\
\hline & 33 & 13 & 0.724 & 1.355 & 40 & 1.463 & -0.036 \\
\hline
\end{tabular}

aThis is the distance between the iris and the edge of the IOL.

bThis is the distance between the posterior cornea and the edge of the IOL. 


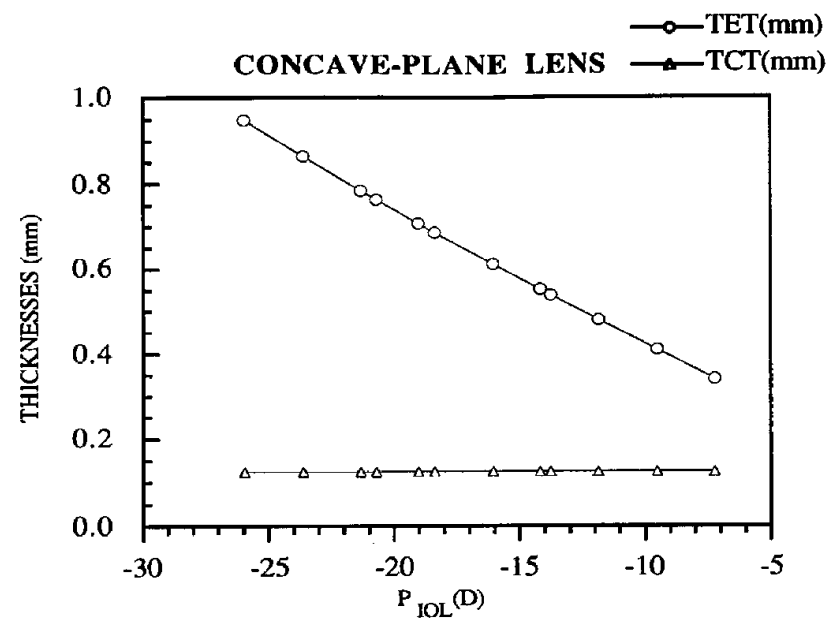

Fig. 7. Graph of edge and central thicknesses as a function of the corrective power of the concave-plane lens (TET, total edge thickness; TCT, total central thickness).

it. The edge of the lens is separated by an average of $0.8 \mathrm{~mm}$ from the iris and $1.4 \mathrm{~mm}$ from the corneal endothelium. These distances are big enough to avoid contact with the aforementioned structures. ${ }^{11,12,18}$ With the concave-plane lens the edge thickness is between $14 \%$ and $41 \%$ of the dimensions of the peripheral anterior chamber (Table 4), and therefore the space between the edges of the lens and the surrounding structures is very small. We considered the lens to be placed in the center of the anterior chamber in all cases (to maintain the same conditions as for the triplet lens), and therefore the distance between the edge of the lens and the iris remains constant: $1.4625 \mathrm{~mm}$. If we vary the power of the lens, the edge thickness would change as well. Therefore, the distance from the edge of the lens to the endothelium, which in all cases is less than $1 \mathrm{~mm}$ (Table 4) and in the majority of the cases is even lower than $0.5 \mathrm{~mm}$, would cause damage to the corneal endothelium. For a lens with a power of $-25.96 \mathrm{D}$ we have no choice but to truncate the lens to insert it

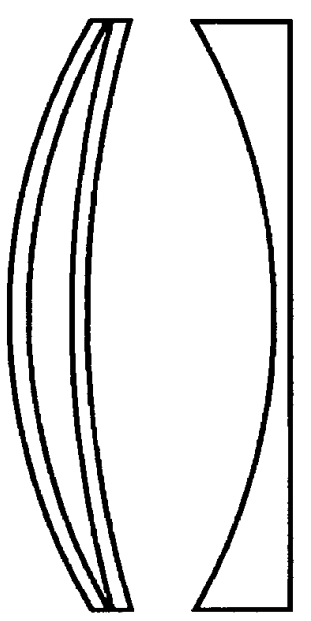

Fig. 8. Design of both triplet and concave-plane lenses and a comparison of their geometries. in the center of the anterior chamber, because its dimensions are bigger than those of the peripheral anterior chamber.

According to Mimouni et al., ${ }^{11}$ the average distance between the concave-plane lens and the iris is 1.118 $\mathrm{mm}$. Therefore, if wetake concave-planelenses with the characteristics that are given in Table 5 and place them at this distance from the iris, the distance between the edge of the lens and the endothelium would increase $0.3445 \mathrm{~mm}$. But even with this movement the distance between the edge of the lens and the endothelium would still be less than $1 \mathrm{~mm}$, except for a lens of $-7.25 \mathrm{D}$. Additionally we would have to remember that if we want to place the lens closer to the iris then the emmetropic power would have a higher absolute value, which would cause an increase in the edge thickness of the lens and therefore the edge would be closer to the endothelium.

The percentages given in Table 4 are important if we wish to find a relation between the edge thickness of the lens and the space in which it is going to be implanted. However, the most important factors are the real distances between the edge of the lens and the iris and the corneal endothelium, because changes in these structures can take place that depend on these distances.

Figure 8 gives a scale representation of a design sample of these two types of IOL as a way to compare their dimensions and shapes. The design of the triplet is clearly closer to the shape of the anterior

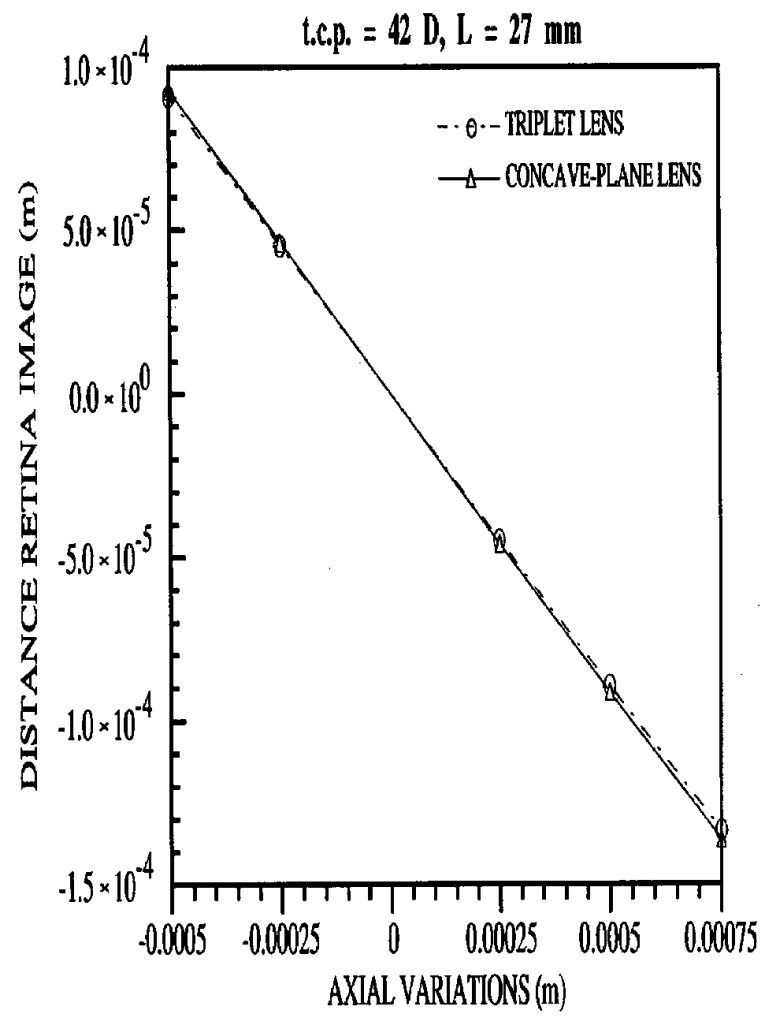

Fig. 9. Graphic representation of the variation in image position as a function of axial displacement for the two types of lenses analyzed (t.c.p., total corneal power; L, axial length). 


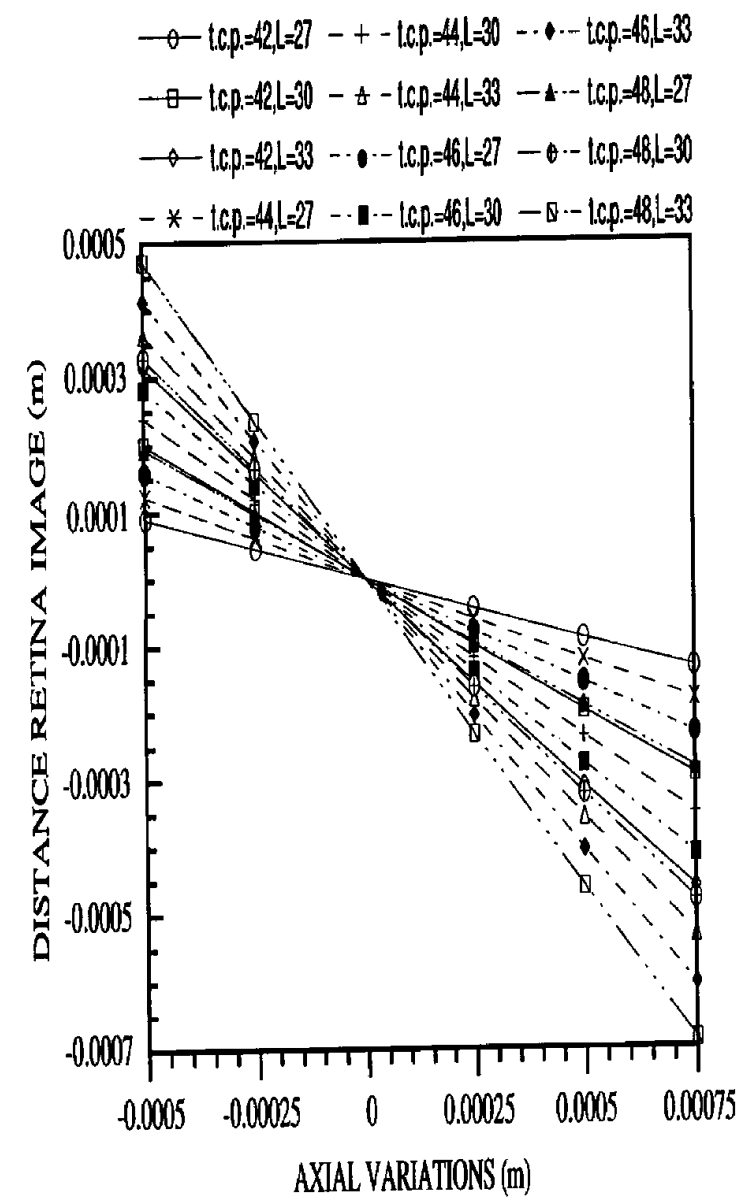

Fig. 10. Variation in image position as a function of axial displacement for the 12 cases analyzed of total corneal power (t.c.p.) and axial length $(\mathrm{L})$ for the triplet lens.

chamber and thus has advantages over the other, because it not only has a lower edgethickness but also has less prominent borders.

\section{Axial Displacement}

Another important point in the analysis of the behavior of an IOL is the study of the variation in position of the image when the lens is not implanted in the right spot or when it moves axially toward the cornea or the crystalline. The calculation of this variation with respect to the position of the retina is shown in Fig. 9 for the two lenses that were studied, the triplet lens and the concave-plane lens, for a keratometric power of $42 \mathrm{D}$ and an axial length of $27 \mathrm{~mm}$. Figure 10 shows us the evolution of this variation in image position for a triplet lens with axial displacement for the 12 theoretical eyes analyzed. This variation is practically the same as the one we find for the concave-plane lens. As we can see, the behavior of the variation in image position with respect to the retina is the same for both types of lens and it depends on the power of the lens that is going to be implanted. The higher the power, the higher the variation in image position.

\section{Discussion and Results}

The implantation of concave-plane IOL's in phakic eyes for the correction of myopia is a technique that gives optical results that are far superior to those obtained through refractive corneal surgery. ${ }^{7}$ However, the problem that concave-planelenses cause is that because of the thickness of the edge and because of prominent edges and geometry, contact between the edges of the lens and the corneal endothelium takes place; this provokes significant morphologic endothelial changes. The solution that we analyzed is a triplet IOL, which has an air chamber in the middle. Here we presented the values of lens power, central thickness, edge thickness, and lens position for the triplet lens designed to correct high myopia and for the concave-plane lens.

The dimensions of the triplet lens compared with those of the concave-plane lens show that if the central thickness is greater in the triplet lens than in the simple concave-plane lens, then the edge thickness will be clearly lower. Therefore the new triplet design will permit a lower edge thickness, which in turn will permit a greater distance to be achieved between the lens and the surrounding structures; thus the possibility of contact between the edge and the endothelium will be greatly reduced. Furthermore, the triplet's geometry adapts perfectly to the anterior chamber and its edges are less prominent, this also contributes to decreasing the risk of contact with the endothelium and consequently the risk of endothelial damage. For these reasons this new design for the triplet IOL is a good alternative for correcting high myopia in phakic eyes, because it avoids the problem of endothelial alterations caused by the lenses that are currently being implanted.

Furthermore, the number of surfaces and the materials that can be combined in the case of the triplet lens increases the possibility of making systems that are also achromatic. ${ }^{19}$ Finally, it should be pointed out that, although this analysis was done on the implantation of a lens in the anterior chamber of eyes with high myopia, the triplet lens can also provide a good solution for correction in the posterior chamber. In such cases the restriction on central and edge thicknesses would not be so strict and different types of lenses could be designed for the same eye, thereby optimizing the design to minimize the aberrations and improve the image quality.

\section{Appendix A}

At a spherical surface between media $\mathrm{n}$ (on the left) and $n^{\prime}$ (on the right) with radius of curvature $r$, the paraxial refraction matrix is

$$
M_{\text {refraction }}=\left[\begin{array}{cc}
1 & 0 \\
\frac{n^{\prime}-n}{n^{\prime} r} & \frac{n}{n^{\prime}}
\end{array}\right] .
$$

The translation operation that transforms a ray from the right side of one optical element through a homogeneous space to the left of a second optical 
el ement can be written in the matrix form as

$$
M_{\text {translation }}=\left[\begin{array}{cc}
1 & -d \\
0 & 1
\end{array}\right],
$$

where $d$ is the distance from the first element to the second el ement.

Appendix B: Symbols Used in This Paper

The following abbreviations and descriptive terms are used in this paper.

\begin{tabular}{|c|c|}
\hline$r_{1}$ & 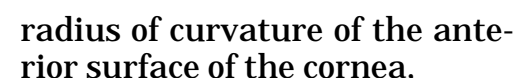 \\
\hline$r_{3}$, & ea, \\
\hline$r_{5}$ & ure of the fir \\
\hline$r_{7}$, & $\begin{array}{l}\text { ure of the sec } \\
\text { elOL, }\end{array}$ \\
\hline$r_{9}$, & the third \\
\hline 11. & the fourth \\
\hline$r_{13}$ & int 20 \\
\hline$r_{15}$ & ste- \\
\hline $\mathrm{n}_{0}$ & \\
\hline$n_{2}$ & \\
\hline$n_{4}$, & \\
\hline $\mathrm{n}_{6}$ & anterior \\
\hline$n_{8}$, & \\
\hline$n_{10}$ & rior \\
\hline 12, & \\
\hline $\mathrm{n}_{14}$, & \\
\hline $\mathrm{n}_{16}$ & indry of t \\
\hline$P_{1}$, & of the anterior surfa \\
\hline$P_{3}$, & posterior surfa \\
\hline$P_{5}$ & e first su \\
\hline$P_{7}$ & e seconc \\
\hline$P_{9}$ & $\begin{array}{l}\text { power of the third surface } \\
\text { IOL, }\end{array}$ \\
\hline$P_{3}$ & e fo \\
\hline$D_{13}-19$ & \\
\hline
\end{tabular}

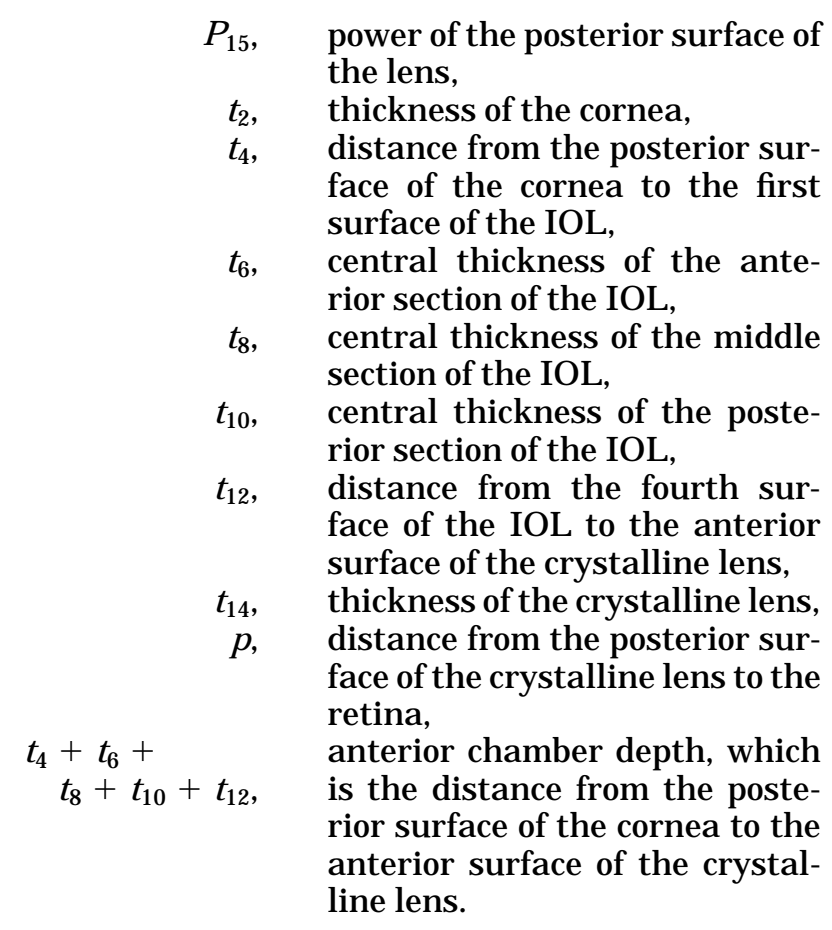

\section{References}

1. J . L. Alioy Sanz, A. Fimia, and I. Pascual, "Lente intraocular," ES patent $P 9,201,253$ (17 J une 1992).

2. W. B. Isaacson, J. A. Futhey, K. D. Thompson, and P. T. I hn, "Multifocal diffractive ophthalmic lens and method of manufacture,"U.S. patent 5,152,788 (6 October 1992).

3. J. P. Colliac, "Matrix formula for intraocular lens power calculation," Invest. Ophthalmol. Vis. Sci. 31, 374-381 (1990).

4. A. Fimia, J. L. Alió, I. Pascual, and A. Beléndez, “New theoretical matrix formula for intraocular lens calculation using the optimal bending factor," J . Cat. Refract. Surg. 19, 293-297 (1993).

5. J . A. Retzlaff, D. R. Sanders, and M. Kraff, Lens I mplant Power Calculation (Slack, Thorofare, N.J ., 1990).

6. Y. Le Grand and S. G. EI Hage, Physi ol ogical Optics (SpringerVerlag, New York, 1980), Chap. 4.

7. G. Baikoff and P. J oly, "Comparison of minus power anterior chamber lenses and myopic epikeratoplasty in phakic eyes," Refract. Corneal Surg. 6, 252-260 (1990).

8. P. J oly, G. Baikoff, and P. Bonnet, "Mise en place d'un implant négatif de chambre antérieure chez des sujets phakes," Bull. Soc. Ophthalmol. Fr. 5, 727-733 (1989).

9. P. P. Choyce, "The correction of high myopia," Refract. Corneal Surg. 8, 242-245 (1992).

10. G. O. Waring, "Phakic intraocular lenses for the correction of myopia-where do we go from here?," Refract. Corneal Surg. 7, 275-276 (1991).

11. F. Mimouni, J . Colin, V. Koffi, and P. Bonnet, "Damage to the corneal endothelium from anterior chamber intraocular lenses in phakic myopic eyes," Refract. Corneal Surg. 7, 277-281 (1991).

12. J . J. Saragoussi, J . Cotinat, G. Renard, M. Savoldelli, A. Abenhaim, and Y. Pouliquen, "Damage to the corneal endothelium by minus power anterior chamber intraocular lenses," Refract. Corneal Surg. 7, 282-285(1991).

13. K. Naeser and E. V. Naeser, "Calculation of the thickness of an intraocular lens,"J . Cat. Refract. Surg. 19, 40-42 (1993).

14. J. T. Holladay, T. C. Prager, T. Y. Chandler, K. H. Musgrove, J. W. Lewis, and R. S. Ruiz, "A three-part system for refining 
intraocular lens power calculations,"' J . Cat. Refract. Surg. 14, 17-24 (1988).

15. J . A. Retzlaff, D. R. Sanders, and M. C. Kraff, "Development of the SRK/T intraocular lens implant power calculation formula,"J . Cat. Refract. Surg. 16, 333-340 (1990).

16. J. T. Holladay, "Intraocular lens power calculation," J . Cat. Refract. Surg. 14, 452-453(1988).
17. A. G. Bennett and R. B. Rabbetts, Clinical Visual Optics (Butterworth, London, 1989), Chap. 2.

18. G. L. Van der Heijde, "Some optical aspects of implantation of an IOL in a myopic eye," Eur. J . Implant Refract. Surg. 1, 245-248 (1989).

19. R. E. Bedford and G. Wyszecki, "Axial chromatic aberration of the human eye,"J . Opt. Soc. Am. 47, 564-565 (1957). 